\title{
Computer Vision and Image Analysis based Techniques for Automatic Characterization of Fruits - a Review
}

\author{
Jyoti A Kodagali \\ Tontadarya College of Engg., Gadag. \\ India.
}

\author{
S Balaji \\ City Engg., College, Bangalore. India
}

\begin{abstract}
This paper presents the recent developments and application of image analysis and computer vision system in an automatic fruit recognition system. It is essential to throw light on basic concepts and technologies associated with computer vision system, a tool used in image analysis of fruit characterization. In India, in view of the ever-increasing population, losses in handling and processing and the increased expectation of food products of high quality and safety standards, there is a need for the growth of accurate, fast and objective quality determination of fruits. A number of challenges had to be overcome to enable the system to perform automatic recognition of the kind of fruit or fruit variety using the images from the camera. Several types of fruits are subject to significant variation in color and texture, depending on how ripe they are. There are many processes in agriculture where decisions are made based on the appearance of the product. Applications for grading the fruit by its quality, size or ripeness are based on its appearance, as well as a decision on whether it is healthy or diseased. The objective of this paper is to provide in depth introduction of machine vision system, its components and recent work reported on an automatic fruit characterization system.
\end{abstract}

\section{General Terms}

Image analysis, fruit characterization, computer vision.

\section{INTRODUCTION}

Agriculture is Indian economy's mainstay and it contributes $18.5 \%$ of the gross domestic products [1]. Due to the advance of cultivation technology, the total cultivation areas and yields for agricultural products have increased rapidly in recent years, generating tremendous market values. Though this suggests vast potential for India to emerge as a major exporter of agricultural produce, its share in global market is very low due to very high post harvest losses in handling and processing, mismanagement of trades and procurements, lack of knowledge of preservation and quick quality evaluation techniques. Ensuring product recognition and quality is one of the most important and challenging tasks of the industry before exporting food and agricultural produce. The Computer vision is a rapid, economic, consistent and objective inspection technique, which has expanded into many diverse industries.

\section{COMPUTER VISION}

Computer vision is a relatively young discipline with its origin traced back to the 1960s [2]. Following an explosion of interest during the 1970s, it has experienced continued growth both in theory and application. Computer vision is considered study and application of methods which allows computers to examine and extract image contents or content of multidimensional data in general to facilitate solving a specific vision problem, such as pattern classification problem [3]. There are six main areas of computer vision: sensing, preprocessing, segmentation, description, recognition, and interpretation. Examples of computer vision applications include systems for controlling processes such as an industrial robot or an autonomous vehicle. Another example is detecting events for visual surveillance or organizing information for indexing databases of images and image sequences. Modelling objects or environments such as medical image analysis or topographical modelling is another use of computer vision. Computer vision system is also applied for agriculture issues such as a system for monitoring crops growth and weeds under rain shelter or a computer vision system for detecting weeds in cereal crops. The recognition system can be applied for educational purpose to enhanced learning, especially for small kids and Down syndrome patients, of agricultural and food products pattern recognition. It can be used in grocery store which makes the customers label their purchases using automatic recognition based on computer vision.

Computer vision is the construction of explicit and meaningful descriptions of physical objects from images. Recognition system has emerged as a 'grand challenge' for computer vision, with the long term aim of being able to achieve near human levels of recognition for tens of thousands of categories under a wide variety of conditions. It is central to optical character recognition, voice recognition, and handwriting recognition. It uses methods from statistics, machine learning and other areas. Typical applications are text classification to recognize different types of texts such as spam and non spam E-mails, speech recantation for specified purposes such as translating different languages to English, hand written recognition for postal codes, or the automatic face recognition which deals with digital images as input to the pattern recognition systems. Several types of image analysis techniques are applied to analyze the agricultural images such as fruits and vegetables, for recognition and classification purposes.

TABLE 1. APPLICATION OF COMPUTER VISION IN DIFFERENT AREAS

\begin{tabular}{|c|c|}
\hline Application Area & Purpose \\
\hline $\begin{array}{c}\text { Industrial automation and } \\
\text { image processing }\end{array}$ & $\begin{array}{c}\text { Process control, quality } \\
\text { control, geometrical } \\
\text { measurement, } \\
\text { Barcode and package label } \\
\text { reading, object sorting } \\
\text { Parts identification on } \\
\text { assembly lines, defect and } \\
\text { fault inspection }\end{array}$ \\
\hline
\end{tabular}




\begin{tabular}{|c|c|}
\hline & $\begin{array}{l}\text { Inspection of printed circuit } \\
\text { boards and integrated circuits }\end{array}$ \\
\hline Medical image analysis & $\begin{array}{l}\text { Tumor detection, } \\
\text { measurement of size and } \\
\text { shape of internal organs, } \\
\text { blood cell count } \\
\text { X-ray inspection }\end{array}$ \\
\hline Robotics & $\begin{array}{l}\text { Obstacle avoidance by } \\
\text { recognition and interpretation } \\
\text { of objects in a scene } \\
\text { Collision avoidance, } \\
\text { machining monitoring, } \\
\text { Hazard determination }\end{array}$ \\
\hline Radar imaging & $\begin{array}{c}\text { Target detection and } \\
\text { identification, guidance of } \\
\text { helicopters and aircrafts in } \\
\text { landing, guidance of remote } \\
\text { piloted vehicles (RPV), } \\
\text { guiding missiles and satellites } \\
\text { from visual cues }\end{array}$ \\
\hline Food industry & $\begin{array}{l}\text { Sorting of vegetables and } \\
\text { fruits, location of defects e.g. } \\
\text { location of dark contaminants } \\
\text { and insects in cereals }\end{array}$ \\
\hline Document analysis & $\begin{array}{l}\text { Handwritten character } \\
\text { recognition, layout } \\
\text { recognition, graphics } \\
\text { recognition }\end{array}$ \\
\hline
\end{tabular}

Singh et al. (2004) [4]

\section{COMPONENTS OF COMPUTER VISION}

A computer vision system generally consists of five basic components: lighting system, a camera, an image capture board (frame grabber or digitizer), personal computer and software as shown in Fig 1.

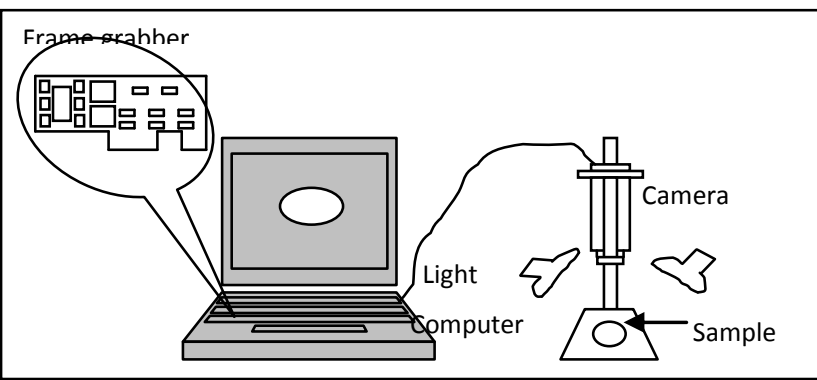

Fig 1: Components of a Computer Vision System
The lighting system must be designed carefully to provide consistent picture eliminating the appearance of variations. The primary key value for lighting is its sources, arrangements and lighting geometry. The factors that influence the selection in lighting is whether the object under inspection is: 1) flat or curved; 2) absorbing, transmissive or reflective; and 3) the nature of the feature to be imaged in comparison with the background. Lighting arrangements are grouped into front- or back-lighting [1]. Front lighting serve as illumination focusing on the object for better detection of external surface features of the product while back-lighting is used for enhancing the background of the object. Light sources used include incandescent lamps, fluorescent lamps, lasers, X-ray tubes and infra-red lamps.

Image capturing is an application program that enables users to upload pictures from digital cameras or scanners which are either connected directly to the computer or to the network. Selection of the frame grabber is based on the camera output, spatial and grey level resolutions required, and the processing capability of the processor board itself.

The process of converting pictorial images into numerical form is called digitization. In this process, an image is divided into a two dimensional grid of small regions containing picture elements defined as pixels by using a vision processor board called a digitizer or frame grabber.

A personal computer or microprocessor system is used to provide disk storage of images and computational capability with vendor supplied software and specific application programs. Also, the system is provided with a high-resolution colour monitor, which aids in visualizing images and the effects of various image analysis routines.

\section{IMAGE PROCESSING TECHNIQUES}

Image processing and analysis techniques are mainly categorized into three levels: high level processing (recognition and interpretation), intermediate level processing (image segmentation and image representation and description) and low level processing (image acquisition and pre-processing).

\subsection{Image Acquisition}

Image capturing devices or sensors are used to view and generate images of the samples. Some of the devices or sensors used in generating images include scanners, ultrasound, X-ray and near infrared spectroscopy. However, in machine vision, image sensors used are the solid state charged coupled device (CCD) (i.e. camera) technology with some applications using thermionic tube devices. Recent technology has seen the adoption of digital camera, which eliminates the additional component required to convert images taken by photographic and CCD cameras or other sensors to readable format by computer processors. Images captured or taken by digital camera maintain the features of the images with little noise due to its variable resolution. 


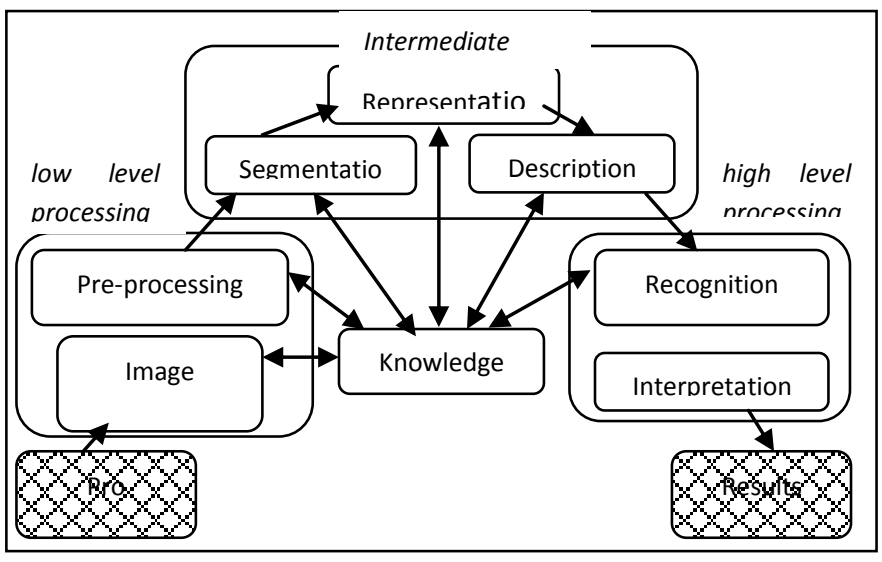

Fig 2: Different Levels in Image Processing

\subsection{Image Preprocessing}

This refers to the initial processing of the raw image. The images captured or taken are transferred onto a computer and are converted to digital images. Digital images though displayed on the screen as pictures, are digits, which are readable by the computer and are converted to tiny dots or picture elements representing the real objects. In some cases, pre-processing is done to improve the image quality by suppressing undesired distortions referred to as "noise" or by the enhancement of important features of interest. The images or pictures are transformed into computer digital readable format (i.e. digitized) if a digital camera did not take them by the image board digitizer. The digitized format is then transferred and used as the input data by the image processing software to carry out the necessary processes. Each or a combination of the digits represent a small portion of the image called picture element (pixel). Objects are described as black and white pictures which are represented by digits ranging from 0 to 255 where 0 is black and 255 is white. Each pixel in coloured pictures is represented by 3 digits representing RGB [Red, Green, Blue] components with each being ( 0 to 255 ) darkest to lightest RGB. An arrangement of these digits in row-column format gives a representation of the image. With this arrangement, image analysis can be done using matrix theory and other mathematical techniques.

\subsection{Segmentation}

Image segmentation is a process of cutting, adding and feature analysis of images aimed at dividing an image into regions that have a strong correlation with objects or areas of interest using the principle of matrix analysis. Segmentation can be achieved by the following techniques: thresholding, edge based segmentation and region based segmentation. Thresholding is used in characterizing image regions based on constant reflectivity or light absorption of their surface. This shows that regions with same features are characterized and extracted together. Thresholding is a process where only the dark region is of interest, the other regions are converted to the background colour in the threshed image before further processing such as sending signals to a device to take a feature based decision. This process is useful in colour (maturity) and feature based (defect and damages detection) sorting.

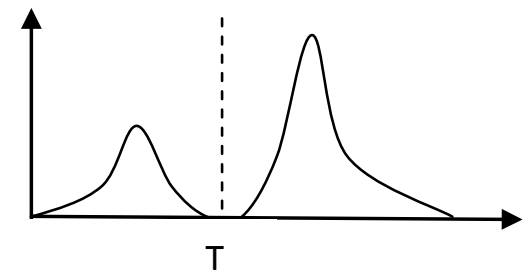

Fig 3: Thresholding

Edge based segmentation relies on detection by edge-to-edge operators, which detect discontinuities in grey level, the pixel, colour, texture etc. Edge detection is useful in shape and size sorting. An example of edge detection result is shown in Fig 4. The application of this process was reported by Raji et al [3] who demonstrated the detection of defects in the shape of bread and biscuit samples on a processing line.
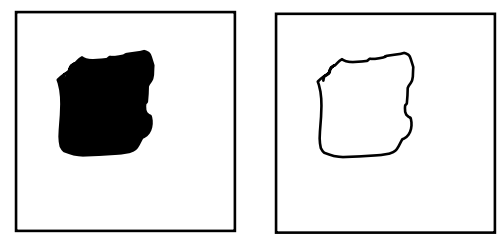

Fig 4: Edge Based Segmentation

Region based segmentation involves the grouping together and extraction of similar pixels to form regions representing single objects within the image as shown in Fig 5. In this process, the other regions are deleted leaving only the feature of interest.

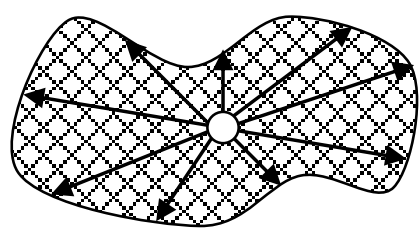

Fig 5: Region Based Segmentation

High level processing deals with recognition and interpretation of images using statistical classifiers or multiplayer neural networks of region of interest. Digital morphology is a group of mathematical operations that can be applied to the set of pixels to enhance or highlight specific aspects of the shape so that they can be counted or recognized. Texture effectively describes the properties of elements constituting the object surface, thus the texture measurements are believed to contain substantial information for the pattern recognition of objects. The repetition of a pattern or patterns over a region is called texture. This pattern may be repeated exactly, or as set of small variations. Texture has a conflictive random aspect: the size, shape, color, and orientation of the elements of the pattern (textons). Although texture can be roughly defined as the combination of some innate image properties, including fineness, coarseness, smoothness, granulation, randomness, lineation, hummocky, etc., a strictly scientific definition has still not been determined. 
Accordingly, there is no ideal method for measuring textures [1].

These steps provide information necessary for the process or machine control for quality sorting and grading. An interaction of all these levels and knowledge database are very important and essential for more precise decision-making and is seen as an integral part of the image processing process. These theories when applied to images of products taken can be used to extract features, which are needed for the necessary processes. Generally, edge detection to determine shape and feature extraction to determine differences in colour is useful in identification and classification

\section{COMPUTER VISION INAGRICULTURE}

The use of computers to analyze images has many potential applications for automated agricultural tasks. But, the variability of the agricultural objects makes it very difficult to adapt the existing industrial algorithms to the agricultural domain. The agricultural systems must support this flexibility, and methods for including domain knowledge in algorithms should be studied as a rational way to cope with this variability. There are many processes in agriculture where decisions are made based on the appearance of the product. Applications for grading the fruit by its quality, size or ripeness are based on its appearance, as well as a decision on whether it is healthy or diseased. Humans are easily able to perform intensive tasks like harvesting and pruning using basically the visual sensory mechanism. This suggests that a system based on a visual sensor should be able to emulate the interpretation process of the human visual recognition system.

A number of challenges had to be overcome to enable the system to perform automatic recognition of the kind of fruit or vegetable using the images from the camera. Many types of vegetables, grains, fruits are subject to significant variation in color and texture, depending on how ripe they are. For example, bananas range from being uniformly green, to yellow, to patchy and brown. The fruit and vegetable market is getting highly selective, requiring their suppliers to distribute the goods according to high standards of quality and presentation. Recognizing different kinds of vegetables and fruits is a recurrent task in supermarkets, where the cashier must be able to point out not only the species of a particular fruit (i.e., banana, apple, pear) but also its variety (i.e., Golden Delicious, Jonagold, Fuji), which will determine its price. Large number of products are handled and sold at the same time. These products consist of ripe and unripe fruits. The introduction of classification of products will encourage the sorting of the unripe (which can be kept for a relatively longer period) from the ripe, which are to be sold immediately. Presently the practice at the fruit market is to sell baskets of fruits containing both ripe and unripe. These products are found to get spoilt before they get to the final destination. With the increasing nature of computer vision and image analysis, there is need for increased computer capabilities and greater processing speed of algorithms to meet the necessary online speeds. To maintain the freshness and the attractiveness of agricultural produce for a longer time this technology needs to be employed.

Besides the progress in research, there is increasing evidence of computer vision systems being adopted at commercial level. This is indicated by the sales of ASME (Application Specific Machine Vision) systems into the North American food market, which reached 65 million dollars in 1995 [6]. Gunasekaran [5] reported that the food industry is now ranked among the top ten industries using machine vision technology. This paper reviews the latest development of computer vision technology with respect to identification, classification and quality inspection in the automatic fruit recognition system.

\section{ASSESSMENT OF FRUITS}

Computer vision has been widely used for the quality inspection and grading of fruits and vegetables. Computer vision is generally used for grading, defect detection, classification and finding out the ripeness of fruits based on their appearance.

\subsection{Apples}

The study of apples using computer vision has attracted much interest and reflects the progress of computer vision technology for fruit inspection.

P. Sudhakara Rao et al. [7] have adopted HSI model for sorting and grading of fruits by colour and developed a prototype for on-line sorting of Apples based on colour, size and shape. The basic image capturing system adopted by them is a colour CCD camera and a frame grabber card and it provided the image in RGB model. The RGB model, after normalisation, is first converted into HSI model using a set of converting equations. The image is analyzed by using advanced image processing algorithms to estimate the colour of image. By representing median density of Hue as a grading criterion, the image processing system achieved around $98 \%$ accuracy in colour inspection of apples.

V. Leemans et al [8] have graded apples into four classes according to European standards. Two varieties were tested: Golden Delicious and Jonagold. The image database included more than a 1000 images of fruits (528 Golden Delicious, 642 Jonagold) belonging to the three acceptable categories \{Extra, I and II $\}$ and the reject (each class represents, respectively, about 60,10 and $20 \%$ of the sample size).The image grading was achieved in six steps: image acquisition; ground colour classification; defect segmentation; calyx and stem recognition; defects characterisation and finally the fruit classification into quality classes. The proposed method for apple external quality grading showed correct classification rates of $78 \%$ and $72 \%$, for Golden Delicious and Jonagold apples, respectively. The healthy fruits were better graded and an error rate which drops to $5 \%$ and $10 \%$, respectively.

P. Sudhakara Rao et.al. [9] have developed six different methods to determine the size of this category of apples by applying the known geometrical models such as circle method, parabola method, ellipse method, principal axis 
method, radius and area signature method and coefficient of variation method.

H. Mirzaei and others [10] have proposed an algorithm to detect the apple skin bruises by applying a threshold and a few morphological operations such as opening and closing. The result of this experiment showed that False Alarm (FA) and Detection Rate (DR) were respectively equal to $14 \%$ and $79.5 \%$.

D. Stajnko and others [11] have proposed a fruit detection algorithm to estimate the diameter and number of apple fruits on a tree. Fruit detection was sufficiently accurate with an 89 $\%$ rate and an overall error rate of $2.2 \%$.

Leemans [12] investigated the defect segmentation of “ Golden Delicious" apples using machine vision. To segment the defects, each pixel of an apple image was compared with a global model of healthy fruits by making use of the Mahalanobis distances. The proposed algorithm was found to be effective in detecting various defects such as bruises, russet, scab, fungi or wounds.

Devrim Unay et al [13] present an application work for grading of apple fruits by machine vision. Here precise segmentation of defects by minimal confusion with stem/calyx areas on multispectral images, statistical, textural and geometric features are extracted from the segmented area. Using these features, statistical and syntactical classifiers are trained for two- and multi-category grading of the fruits and achieved recognition rates of $93.5 \%$.

M.B. Lak et al [14] have developed edge detection based algorithm and color-shape based algorithm to segment images of red apples obtained under natural lighting. Edge detection based algorithm was not successful, while color-shape based algorithm could detect apple fruits in $83.33 \%$ of images.

Brendon J. Woodford and others [15] have detailed the image processing, and neural network classification methods like neural network classifier using wavelets applied to the task of identifying the pest that caused the damage to apple fruits and leaves in orchards. They have obtained the high classification rate on a standard neural network without any special alteration to the learning algorithm.

Ismail Kavdir [16] haas applied Fuzzy logic (FL) as a decision making support to grade apples. Quality features such as the color, size and defects of apples were measured through different equipment. The same set of apples was graded by both a human expert and a FL system designed for this purpose. Grading results obtained from FL showed 89\% general agreement with the results from the human expert, providing good flexibility in reflecting the experts expectations and grading standards into the results.

Devrim Unay [17] has proposed a method for apple defect detection and quality classification with MLP-neural networks. Here, the initial analysis of a quality classification system for 'Jonagold' and 'Golden Delicious' apples were shown. Later, color, texture and wavelet features are extracted from the apple images. Principal components analysis was applied on the extracted features and some preliminary performance tests were done with single and multi layer perceptrons. The best results were 89.9 and 83.7 per cent for overall and defected pixels of 6 defected images.

Devrim Unay et al [18] present several thresholding and classification-based techniques that were employed for pixelwise segmentation of surface defects of 'Jonagold' apples. Here, the Segmentation by supervised classifiers was the most accurate, and the average of class-specific recognition errors was more reliable than error measures based on defect size or global recognition. Segmentation accuracy was improved when pixels were represented as a neighbourhood. The effect of down-sampling on segmentation accuracy and computation times showed that multi-layer perceptrons were the best. Russet was the most difficult defect to segment, and flesh damage the least. The proposed method was much more precise on healthy fruit.

\subsection{Dates}

Yousef Al Ohali [19] has designed and implemented a prototype computer vision based date grading and sorting system. They have defined a set of external quality features such as flabbiness, size, shape, intensity and defects. The system used RGB images of the date fruits and from these images, it automatically extracted the afore-mentioned external date quality features. Based on the extracted features it classified dates into three quality categories (grades 1,2 and 3) defined by experts using back propagation neural network classifier and tested the accuracy of the system on preselected date samples. The test results showed that the system can sort $80 \%$ dates accurately.

\subsection{Grapes}

Dae Gwan Kim [20] has designed techniques of color imaging and texture feature analysis that are used for classifying citrus peel diseases under the controlled laboratory lighting conditions. A total of 39 image texture features were determined from the transformed hue $(\mathrm{H})$, saturation $(\mathrm{S})$, and intensity (I) region-of-interest images using the color cooccurrence method for each fruit sample. The model using 14 selected HSI texture features achieved the best classification accuracy $(96.7 \%)$, which suggested that it would be the best to use a reduced hue, saturation and intensity texture feature set to differentiate citrus peel diseases. Average classification accuracy and standard deviation were $96.0 \%$ and $2.3 \%$, respectively, for a stability test of the classification model, indicating that the model is robust for classifying new fruit samples according to their peel conditions.

\subsection{Peach}

A. Esehaghbeygi et al [21] have introduced the machine vision system for evaluation and classification of the Iranian saffron peach. Physical features such as size and colour were measured to categorize peaches into three quality classes of red-yellow, yellow-red, and yellow. The HIS model hue, 
saturation, and luminance was used for colour processing of flawless samples and four boundaries were selected for the peach size image. The colours of peaches estimated by the system were correlated with the colorimetric index values that are currently used as standards. Experimental results using the USDA standard, the size classification accuracy achieved is $96 \%$, while the colour classification accuracy is $90 \%$, and that the spot detection algorithm performs well with correct detection levels of $97 \%$ and $85 \%$ for brown and white skin spots, respectively.

\subsection{Jatropha}

Zulham Effendi et al [22] have developed a Grading System of Jatropha (GSJ) by using color histogram method to distinguish the level of ripeness of the fruits based on the color intensity.

\subsection{Palm}

Mohd Z. Abdullah et al [23] have used the color vision system HSI (Hue, Saturation and Intensity) color space and applied multivariate discriminant analysis to classify oil palms into four quality grades according to PORIM (Palm Oil Research Institute of Malaysia) inspection standards. These are the unripe, the under ripe, the optimally ripe and the overripe classes. Depending upon the quality features evaluated, misclassification by the vision system varied from 5\% to $12 \%$ but averaged at about $8 \%$. Machine vision disagreement ranged from $2 \%$ to $19 \%$.

\subsection{Pomeogranate}

Jose Blasco et al [24] have developed machine for automatic inspection of pomegranate arils for fresh consumption wherein this machine individualizes, inspects, classifies and separates the arils in four categories. They have used multivariate analysis models to classify the arils with an average success of about $90 \%$.

\subsection{Watermelon}

Hassan Sadrnia et al [25] have classified the long type watermelon depending on the fruit shapes. Physical characteristics of watermelon such as mass, volume, dimensions, density, spherical coefficient and geometric mean diameter were measured. Relations and correlations coefficient were obtained between characteristics for normal and non-standard fruit shape. It was found that weight of normal watermelon could be determined by image analysis with an error of $2.42 \%$.

\subsection{Rasin}

Mahdi Abbasgolipour et al [26] have proposed a method for classification and sorting of raisins where image processing of images is done with an algorithm developed in Visual BASIC 6.0. The accuracy of the proposed system is about $93 \%$.

M Omid et al [27] developed and implemented an efficient algorithm in Visual BASIC language in order to extract useful features of captured images of bulk raisins by image processing technique. The proposed machine vision system consisted of a video camera, a capture card, a lighting adjustable tunnel, a personal computer, and mechanical parts.
The developed algorithm initially extracts the raisins by removing the background from the taken images. It then sorts the raisins according to their RGB color and size features. By a suitable combination of length and RGB color values raisins were graded in two classes. Final step in the algorithm is the calculation of the centre of gravity of each raisin to be later used for automatic sorting and rejection of bad raisins. The proposed system was developed and tested and results showed that its accuracy is about $96 \%$.

\subsection{Bananna}

Yizhong Wang [28] proposed a non-destructive measuring and evaluating method for fruits based on color identification. The color images of fruits were taken and images' RGB histograms were calculated and used as quality parameters for fruits. A BP neural network with three layers was established wherein its input and output were the RGB histograms and evaluating results, respectively. After training, the qualities of fruits were identified by the BP network according to the histogram of RGB of fruits' images. For verifying the proposed method they used the qualities of bananas that were measured and evaluated.

\subsection{Orange}

B Sathya Bama [29] has proposed an approach for quality inspection of orange fruits using combined color texture features. In the proposed method, input image is segmented using histogram based thresholding technique to identify whether the portion of image is defective or non defective. For defective samples, texture features were extracted from $3 \mathrm{D}$ co-occurance distribution. Sum of squared distance (SSD) is calculated between texture features of training and test fruits. Non-defective fruits were considered as extra class while class I and class II were considered as moderately and highly defective fruits, respectively. A database of 150 samples was collected from mozambi and orange fruits with all quality classes for marketable. 80 images were manually classified for training and remaining were used for testing. Classification rate was greater than $93 \%$.

\subsection{Others}

S. Arivazhagan and others [30] proposed an efficient fusion of color and texture features for fruit recognition. The recognition was done by the minimum distance classifier based upon the statistical and co-occurrence features derived from the Wavelet transformed sub-bands. Experimental results on a database of about 2,635 fruits from 15 different classes confirmed the effectiveness of the proposed approach and the successful recognition rate was $86 \%$.

\section{CONCLUSION}

This paper reviewed the recent developments in the computer vision for the automatic fruit recognition system with various characteristics such as defect, quality, color, texture etc. Computer vision systems have been used increasingly in the industry for inspection and evaluation purposes as they can provide rapid, economic, hygienic, consistent and objective assessment. However, difficulties still exist, evident from the relatively slow commercial uptake of computer vision technology in all sectors. Even though adequately efficient 
and accurate algorithms have been produced, processing speeds still fail to meet modern manufacturing requirements. However, the survey reveals that still much of the work needs to be concentrated on the fruits recognition in variety of angles of the fruit. Considering the quantum of work being done and the success rates achived, we can say that computer vision is a powerful tool of automation that includes both image processing and image analysis tools.

\section{REFERENCES}

[1] Krishna Kumar Patel \& A. Kar \& S. N. Jha \& M. A. Khan, February 2011, Machine vision system: a tool for quality inspection of food and agricultural products, $\mathrm{J}$ Food Sci Technol, DOI 10.1007/s13197-011-0321-4.

[2] Baxes GA, 1994, Digital image processing principles and applications, Wiley, New York.

[3] Raji a. O and A. O. Alamutu, February 2005, Prospects of Computer Vision Automated Sorting Systems in Agricultural Process operations in Nigeria, CIGR, Journal of Scientific Research and Development". Vol. VII.

[4] Singh M, Kaur G, Kaur S, 7 Oct 2004, Machine vision: an upcoming realm Everyman's science, 186-187.

[5] Gunasekaran, S., Computer vision technology for food quality assurance, 1996, Trends in Food Science and Technology, 7(8), pp.245-256.

[6] Locht, P., Thomsen, K., Mikkelsen, P., 1997, Full colour image analysis as a tool for quality control and process development in the food industry, Paper No. 973006, ASAE, 2950 Niles Road, St. Joseph, MI 49085-9659, USA.

[7] P. Sudhakara Rao et al, 2009, Colour Analysis of fruits using machine vision system for Automatic Sorting and Grading, J. Instrum. Soc. India 34 (4) 284-291.

[8] V. Leemans et al, 2002, On-line Fruit Grading according to their External Quality using Machine Vision, Biosystems Engineering 83 (4), 397-404, doi:10.1006/bioe.2002.0131,Journal of Automation and Emerging Technologies, Belgium.

[9] P. Sudhakara Rao et al, summer-fall 2002, New Approaches for Size Determination of Apple Fruits for Automatic Sorting and Grading, Iranian Journal of Electrical and computer engineering, vol.1, no. 2.

[10] H. Mirzaei et al, A New and Robust Apple Evaluation Method using Image Processing, 29-31Aug 2007, First Joint Congress on Fuzzy and Intelligent Systems Ferdowsi University of Mashhad, Iran.

[11] D. Stajnko, J. Rakun and M. Blanke, 2009, Modelling Apple Fruit Yield Using Image Analysis for Fruit Colour, Shape and Texture, Europ.J.Hort.Sci., 74 (6). S. 260-267, ISSN 1611-4426.

[12] V. Leemans, M.-F. Destain, A real-time grading method of apples based on features extracted from defects.

[13] Devrim Unay et al, 2010, Automatic grading of Bicolored apples by multispectral machine vision, Journal of Computers and Electronics in Agriculture, elesevier.

[14] M. B. Lak et al, 2010, Apple Fruits Recognition Under Natural Luminance Using Machine Vision, Advance
Journal of Food Science and Technology 2(6): 325-327, ISSN: 2042-4876.

[15] Brendon J. Woodford et al, 2010, Fruit Image Analysis using Wavelets.

[16] Ismail Kavdir et al, 2003, Apple Grading Using Fuzzy Logic.

[17] D. Unay, B. Gosselin, 2006, Automatic defect segmentation of Jonagold apples on multi-spectral images: A comparative study, Postharvest Biology and Technology 42 271-279.

[18] Devrim Unay, Bernard Gosselin, Apple Defect Detection and Quality Classification with MLP-Neural Networks.

[19] Yousef Al Ohali, 2011, Computer vision based date fruit grading system: Design and implementation, Journal of King Saud University - Computer and Information Science 23, 29-36.

[20] Dae Gwan Kim et al, September, 2009, Classification of grapefruit peel diseases using color texture feature analysis, International Journal of Agric \& Biol Eng,, Vol. 2 No.3, USA.

[21] A. Esehaghbeygi et al, 2010, Digital image processing for quality ranking of saffron peach, Int. Agrophysics, 24, 115-120.

[22] Zulham Effendi et al, 2009, Development of Jatropha Curcas Color Grading System for Ripeness Evaluation, European Journal of Scientific Research ISSN 1450216X Vol.30 No.4 , pp.662-669.

[23] Mohd Z. Abdullah et al, 2002, Color Vision System for Ripeness Inspection of Oil Palm Elaeis Guineenszs, Journal of Food Processing Preservation 26 213-235, Malaysia.

[24] Jose Blasco et al, Computer vision developments for the automatic inspection of fresh and processed fruits, Image Analysis for Agricultural Products and Processes, ISSN 0947-7314, Spain.

[25] Hassan Sadrnia et al, Classification and Analysis of Fruit Shapes in Long TypeWatermelon Using Image Processing, International Journal of Agriculture \& Biology 1560-8530/2007/09-1-68-70, Iran.

[26] Mahdi Abbasgolipour, Feb 2010, Sorting Raisins by Machine Vision System,Modern Applied Science, Vol. 4, No. 2.

[27] M. Omid, M. Abbasgolipour, A. Keyhani and S.S. Mohtasebi, 2010, Implementation of an Efficient Image Processing Algorithm for Grading Raisins, International Journal of Signal and Image Processing, Vol.1-Iss.1.

[28] Yizhong Wang et al, 2009, Study on fruit quality measurement and evaluation based on color Identification, Proc. of SPIE Vol. 7513 75130F-1.

[29] B Sathya Bama et al, 2011, 3D Color Co-occurance texture features as tool to evaluate quality of fruits, Journal of Scientific and Industrial Research, Vol 70, pp. 912-917.

[30] S.Arivazhagan et al, Oct 2010, Fruit Recognition using Color and Texture Features, Journal of Emerging Trends in Computing and Information Sciences, Vol. 1, No. 2. 